\title{
Stochastic optimization model of aquacultured fish for sale and ecological education
}

\author{
Hidekazu Yoshioka ${ }^{*}$ and Yuta Yaegashi
}

\section{"Correspondence:}

yoshih@life.shimane-u.ac.jp

${ }^{1}$ Faculty of Life and Environmental

Science, Shimane University,

Nishikawatsu-cho 1060, Matsue,

Shimane 690-8504, Japan

Full list of author information is

available at the end of the article

\begin{abstract}
A stochastic optimization model for management of aquacultured fish for sale and ecological education is established. Population dynamics of the fish is described with a system of stochastic differential equations assuming that they are stochastically harvested after an opening time: the variable to be optimized. A remarkable difference between the present and conventional models for the aquaculture is that the former considers the harvesting for ecological education, which is a key for current inland fishery especially in Japan. Finding the cost-effective optimal opening time effectively reduces to solving an algebraic equation. Mathematical analysis on the optimal opening time clearly reveals its unique existence and qualitative behavior, such as its dependence on the model parameters, which have practical implications. A demonstrative application example of the model is also presented focusing on an aquaculture of Plecoglossus altivelis in Japan.
\end{abstract}

Keywords: aquaculture; population dynamics; stochastic differential equation; Poisson process; fisheries cooperatives; ecological education

\section{Introduction}

Aquaculture is one of the most profitable fisheries sectors that conventionally farms, harvests, and sells fishery resources [1]. Cost-effective and sustainable management strategies for aquaculture have been studied from both scientific and engineering viewpoints. Clarke et al. [2] reviewed fish vaccine development using plant genetic engineering techniques for sustainable aquaculture. Mazid et al. [3] investigated cost-effective feeding strategy for aquacultured fish using locally available ingredients.

Population dynamics models can efficiently simulate behavior and dynamics of aquatic organisms [4-6]. For modeling the dynamics related to aquaculture, stochastic models turned out to be able to compute cost-effective, environmentally- and/or ecologicallysound management strategies of fishery resources under a variety of conditions [7-11]. Stochastic differential equations (SDEs) [12] have served as one of the most important tools for optimization problems in aquaculture because of their power to effectively handle population dynamics subject to uncertainties. Nøstbakken [13] numerically computed optimal management rules of aquaculture systems subject to regime-switching stochastic stocks and prices of farmed fishes. Reed and Clarke [14] derived optimal harvesting and pricing rules for aquacultured fishes with the stochastic and size-dependent growth rate.

(c) The Author(s) 2017. This article is distributed under the terms of the Creative Commons Attribution 4.0 International License (http://creativecommons.org/licenses/by/4.0/), which permits unrestricted use, distribution, and reproduction in any medium, provided you give appropriate credit to the original author(s) and the source, provide a link to the Creative Commons license, and indicate if changes were made. 
León-Santana et al. [15] analytically derived formulae to achieve environmentally-sound aquaculture based on linear SDEs.

Inland fishery resources, many of which are aquacultured, have long been of central importance as food sources of humans [16,17]. Currently, establishment of adequate management policies for aquacultured fishes is an urgent issue for inland Fisheries Cooperatives (FCs) because of their severe financial and operational difficulties, which are considered due to decrease of the fish catches and aging of officers. Recently, the authors proposed deterministic [18] and stochastic [19] optimization models to achieve cost-effective management of artificial aquaculture systems (pools). Their models can find the optimal opening (stopping) time [20], which gives the most cost-effective management strategy of aquacultured fishes. The models, however, lack one important factor in the aquaculture of inland fishery resources that some FCs have to manage harvesting not for sale. The most important purpose of the harvesting not for sale is the ecological education [21, 22]. Actually, each FC in Japan has a duty of ecological and environmental conservation and education around the river where it authorizes [23]. Some FCs in Japan harvest aquacultured fishes for the exchange meetings of ecological education where local residents and their children learn about and enjoy ecology and environment of neighboring rivers [24]. For example, the harvested fishes are used for catching competition of the fishes by the children of local residents in exchange meetings (Figure 1). Through the ecological education, FCs teach about the fishes they farm and aquatic organisms living in their river. Throughout communications between the authors and an FC in Japan during 2016-2017, we found that the effect of ecological education should be considered in optimization models of aquacultured fishes of FCs in Japan, in which fishes are harvested for both sale and ecological education. Furthermore, we also found that FCs consider that the ecological education can be a critical factor to continue inland fishery sustainably. However, to the authors' knowledge, such mathematical modelling of an aquaculture considering the harvesting not for sale, despite its importance, has not been carried out so far. This is the strong motivation for writing this paper.

The purpose of this paper is to develop a new, tractable stochastic process model for aquaculture considering the harvesting not for sale, which can be utilized for decisionmaking of the cost-effective and ecologically conscious aquaculture. Stochastic population dynamics in an artificial aquaculture system is described with a system of SDEs that govern the total number of the population and its representative weight. The opening time of harvesting is set as the variable to be optimized. The decision-maker in our optimization problem is the manager of an FC. The opening time is optimized by the manager so

Figure 1 A catching competition of aquacultured $P$. altivelis by children of local residents. This competition was held in an exchange meeting for ecological education between HRFC and local residents in 2016. This photograph was taken by the first author.

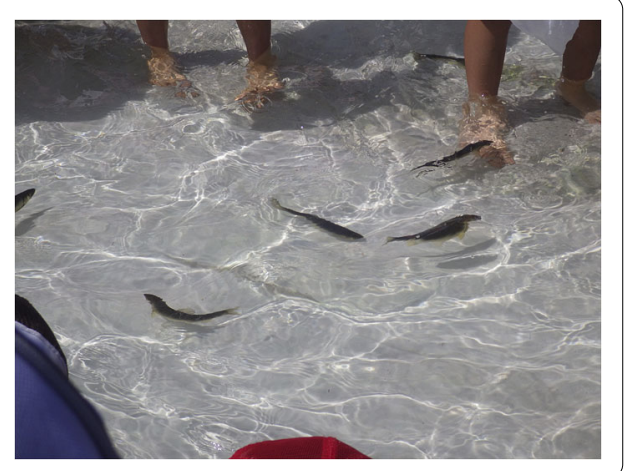


that the performance index, which is a net profit by the aquaculture, is maximized. The main difference between the present and conventional models [18, 25-27] is that fishes are harvested not only for sale but also for ecological education to local residents. This effect is incorporated into both the population dynamics model and the performance index. Mathematical analysis on the optimal opening time is carried out and its results are verified with numerical computation focusing on Plecoglossus altivelis, which is one of the most important inland fishery resources in Japan, managed by Hii River Fisheries Cooperative (HRFC). This paper is the first attempt to model aquaculture considering harvesting for ecological education.

The rest of the paper is organized as follows. Section 2 presents the mathematical model for cost-effective management of aquacultured fishes without reproduction. Section 3 presents mathematical analysis results on the model. Section 4 performs numerical computation to verify the mathematical analysis results with an application to Plecoglossus altivelis (Ayu), which is an important inland fishery resource in Japan. Section 5 concludes this paper and presents future perspectives of our research.

\section{Methods: mathematical model}

The population dynamics model in this paper is not significantly different from the conventional ones [18] except for that it is driven by Poisson noises. On the other hand, the performance index to be maximized, which has a term on profit by harvesting the fish for ecological education, distinguishes the present model from the conventional ones. Hereafter, $a \wedge b$ for $a, b \in \mathbb{R}$ represents the smaller value between $a$ and $b$.

\subsection{Governing equations}

The time is denoted as $t \in[0, T]$ where $t=0, T$ are the initial and terminal times of an aquaculture, respectively. The fish is introduced into the system only at $t=0$. The population dynamics in an artificial aquaculture system is reasonably considered to be nonrenewable [18]. The dynamics is described with the representative (average) weight of individuals $W_{t} \geq 0$ and the total number of individuals $N_{t} \geq 0$. The governing equations of $W_{t}$ and $N_{t}$ are assumed to be independent with each other. This is a reasonable assumption for realistic aquaculture system operated by well-experienced managers with a controlled, sufficiently large pool. The representative weight $W_{t}$ monotonically increases throughout the period. On the other hand, the total number of individuals $N_{t}$ monotonically decreases at the same time. All the stochastic integrals in what follow are defined in the Itô's sense [28].

There is an opening time $\tau \in[0, T]$ of harvesting after which the population is harvested for sale and for exchange meetings for ecological education between FCs and local residents. The exchange meetings are hosted by FCs for ecological education, so that the residents can learn about their fishes and the contents of their works. At each exchange meeting, several hundred individuals of the aquacultured fishes are used for the catching competition by children of local residents: the main event of the meeting (Figure 1). In addition, several lectures on ecology and biology of aquatic organisms including fishes living in and around rivers, lakes, the seas, and in the aquaculture system are held at each meeting. Selling events during $[\tau, T)$ is modelled with a Poisson process $P_{t}^{(1)}$ with the intensity $\lambda^{(1)}>0$. The total number of potentially harvested individuals for sale, the demand, at the time $t$ follows a non-negative continuous-time stationary Markov process $c_{t}^{(1)}$. The 
exchange meetings are held during $[\tau, T)$, which is also modelled as a Poisson process $P_{t}^{(2)}$ with the intensity $\lambda^{(2)}>0$. The total number of potentially harvested individuals for ecological education at the time $t$ follows a non-negative continuous-time stationary Markov process $c_{t}^{(2)}$. Scheduled date and time of each exchange meeting are usually determined before $t=0$, but they are possibly held on different date and time due mainly to climate stochasticity. The mean values of $c_{t}^{(1)}$ and $c_{t}^{(2)}$ are assumed to exist and are denoted as $C^{(1)}>0$ and $C^{(2)}>0$, respectively. The stochastic processes $P_{t}^{(1)}, P_{t}^{(2)}, c_{t}^{(1)}$, and $c_{t}^{(2)}$ are assumed to be independent with each other. The stochastic population dynamics is considered under usual filtration generated by the processes $P_{t}^{(1)}, P_{t}^{(2)}, c_{t}^{(1)}$, and $c_{t}^{(2)}$ as in the conventional stochastic process models. Mathematical properties and applications of Poisson processes are presented in Ross [29].

The initial conditions of $W_{t}$ and $N_{t}$ at the time $t=0$ are assumed to be given as nonnegative numbers. The governing equation of $W_{t}$ is given by

$$
\frac{\mathrm{d} W_{t}}{\mathrm{~d} t}=W_{t} g\left(W_{t}\right), \quad t>0
$$

with the growth rate $g$. This paper assumes the constant $g=r>0$ or the generalized Verhulst model (theta-logistic model) given as [30]

$$
g(w)=r\left(1-\left(\frac{w}{K}\right)^{\theta}\right), \quad w \geq 0
$$

with the intrinsic growth rate $r>0$, the maximum body weight $K>0$, and the nonlinearity parameter $\theta>0$. This model is more realistic than that with $g=r$ since the latter gives unbounded exponential growth. Setting $\theta=1$ in (2) yields the conventional Verhulst model. Behavior of solutions to the generalized Verhulst model and its related models has been investigated in detail [30, 31].

Denote the natural mortality rate of the fish as $R$. The governing equation of $N_{t}$ is given by the SDE

$$
\mathrm{d} N_{t}=-R N_{t} \mathrm{~d} t-\chi_{\{t \geq \tau\}} \sum_{n=1}^{2} c_{t}^{(n)} \mathrm{d} P_{t}^{(n)}, \quad t>0,
$$

which is explicitly solved with stochastic integrals (Chapter 15 of [29]) as

$$
N_{t}= \begin{cases}N_{0} \exp (-R t) & (0 \leq t<\tau), \\ N_{0} \exp (-R t)-\sum_{n=1}^{2} \int_{\tau}^{t} \exp (-R(t-s)) c_{t}^{(n)} \mathrm{d} P_{t}^{(n)} & (\tau \leq t \leq T),\end{cases}
$$

where $\chi_{\{t \geq \tau\}}$ is the indicator function such that $\chi_{\{t \geq \tau\}}=1$ for $t \geq \tau$ and $\chi_{\{t \geq \tau\}}=0$ otherwise. Hereafter, $\mathrm{E}[\cdot]$ represents expectation. The mean $\mathrm{E}\left[N_{t}\right]$ is calculated as

$$
\mathrm{E}\left[N_{t}\right]= \begin{cases}N_{0} \exp (-R t) & (0 \leq t<\tau), \\ \exp (-R(t-\tau))\left[N_{0} \exp (-R \tau)-B(\exp (R(t-\tau))-1)\right] & (\tau \leq t \leq T)\end{cases}
$$

with $B=R^{-1}\left(C^{(1)} \lambda^{(1)}+C^{(2)} \lambda^{(2)}\right)$. 


\subsection{Performance index}

The performance index that quantifies optimality of each management strategy is established from the viewpoint of the manager of an FC as the decision-maker. The performance index consists of (statistically) known variables and parameters so that the decision-maker can choose a strategy before they start an aquaculture. We then assume that the decision-maker can predict the (mean) extinction time $T_{\text {ex }}$ of $\mathrm{E}\left[N_{t}\right]$ :

$$
T_{\text {ex }}=\inf \left\{t \mid E\left[N_{t}\right]=0, t \geq 0\right\} .
$$

This assumption is natural under the situation where the decision-maker can empirically predict laws of the stochastic processes $P_{t}^{(1)}, P_{t}^{(2)}, c_{t}^{(1)}$, and $c_{t}^{(2)}$, but not their paths. It is then reasonable to give the performance index, which is net benefit of the FC by the aquaculture, as an expectation of a functional of stochastic processes. The present performance index assumes a situation where the population is stochastically harvested with fluctuating demands.

The present performance index is formulated as an extended, stochastic counterpart of that in Yoshioka and Yaegashi [18]:

$$
J_{\tau}=\mathrm{E}\left[\int_{\tau}^{T \wedge T_{\mathrm{ex}}} \alpha^{(1)} c_{t}^{(1)} W_{t} \mathrm{~d} P_{t}^{(1)}+\int_{\tau}^{T \wedge T_{\mathrm{ex}}} \alpha^{(2)} c_{t}^{(2)} W_{t} \mathrm{~d} P_{t}^{(2)}-\int_{0}^{T \wedge T_{\mathrm{ex}}} \beta p N_{t} W_{t} \mathrm{~d} t\right]
$$

where $\alpha^{(1)}, \alpha^{(2)}$, and $\beta$ are positive weight parameters $a$ priori determined by the manager, and $p$ is the cost of farming the fish per unit time. As presented in (7), the performance index consists of the three terms. The first term is the profit by selling the fish during the period $[\tau, T]$. The second term is the profit by providing the fish for exchange meetings for ecological education during the period $[\tau, T]$. The second term is possibly difficult to financially evaluate. This term indirectly represents the profit by the exchange meetings. Officers of HRFC consider that harvesting the aquacultured fish for ecological education can indirectly lead to conservation of environment and ecology of rivers by local residents, which consequently becomes beneficial for the FC. Finally, the third term is the cost of farming the fish during the whole period $[0, T]$, which involves feeding cost of the fish and cleaning cost of their excrements in the pool, both of which would be larger for larger total biomass $N_{t} W_{t}$. As described above, $J_{\tau}$ is not purely monetary one whose weights involve the attitude of the decision-maker. Importance of each term can be modulated by the decision-maker based on his/her consideration that which term is of importance for the FC. Therefore, different FCs would have different values of the weight parameters.

Since $J_{\tau}$ is a function of the opening time $\tau \in[0, T]$, and it is continuous with respect to $\tau$, there exists at least one maximizer $\tau=\tau^{*}$ of $J_{\tau}$ such that

$$
\tau^{*}=\underset{\tau \in\left[0, T \wedge T_{\mathrm{ex}}\right]}{\arg \max } J_{\tau}
$$

Remark 2.1 In practice, the orders of $\lambda^{(1)}$ and $\lambda^{(2)}$ are significantly different. As an example of $P$. altivelis farmed by HRFC during 2016, the exchange meetings for ecological education were held at most several times in each month. During the same year, the aquacultured fishes were sold almost every day. Therefore, we infer that $\lambda^{(1)}=O\left(10^{0}\right)(1 /$ day $)$ to $O\left(10^{1}\right)\left(1 /\right.$ day) and $\lambda^{(2)}=O\left(10^{-2}\right)\left(1 /\right.$ day) to $O\left(10^{-1}\right)\left(1 /\right.$ day). We also infer $C^{(1)}=O\left(10^{0}\right)$ to $O\left(10^{1}\right)$ and $C^{(2)}=O\left(10^{2}\right)$ to $O\left(10^{3}\right)$ based on interviews from HRFC during 2016 . 


\section{Mathematical analysis}

Mathematical analysis on existence and uniqueness of the optimal opening time $\tau^{*}$ is carried out. A primitive stochastic process model is also analyzed in this section. Practical implications of the mathematical analysis results are presented as well, which are verified numerically in the next section.

\subsection{Primitive model}

Theoretical behavior of a primitive population dynamics model is briefly studied in order to get insights on the behavior of the presented model. Let $k$ be a fixed natural number. Let $P_{t}^{(n)}(n=1,2, \ldots, k)$ be independent Poisson processes with the intensities of $\lambda^{(n)}>0$. The amplitude of $P_{t}^{(n)}$ is modulated by the stationary and continuous-time non-negative stochastic process $c_{t}^{(n)}$ having the mean value $C^{(n)}$. The processes $P_{t}^{(n)}$ and $c_{t}^{(n)}$ are assumed to be independent with each other. Consider the primitive model that governs a stochastic process $M_{t}$ :

$$
\mathrm{d} M_{t}=-R M_{t} \mathrm{~d} t-\sum_{n=1}^{k} c_{t}^{(n)} \mathrm{d} P_{t}^{(n)}, \quad t>0
$$

The SDE is subject to the initial condition $M_{0}$ at the initial time $t=0$.

The SDE (9) can be solved as

$$
M_{t}=M_{0} \exp (-R t)-\int_{0}^{t} \exp (-R(t-s)) \sum_{n=1}^{k} c_{s}^{(n)} \mathrm{d} P_{s}^{(n)}, \quad t>0
$$

Since $P_{s}^{(n)}$ and $c_{s}^{(n)}$ are independent, the mean $\mathrm{E}\left[M_{t}\right]$ is calculated as

$$
\begin{aligned}
\mathrm{E}\left[M_{t}\right] & =M_{0} \exp (-R t)-\mathrm{E}\left[\int_{0}^{t} \exp (-R(t-s)) \sum_{n=1}^{k} c_{s}^{(n)} \mathrm{d} P_{s}^{(n)}\right] \\
& =M_{0} \exp (-R t)-\int_{0}^{t} \exp (-R(t-s)) \sum_{n=1}^{k} \mathrm{E}\left[c_{s}^{(n)}\right] \lambda^{(n)} \mathrm{d} s \\
& =\exp (-R t)\left[M_{0}-A(\exp (R t)-1)\right], \quad t>0,
\end{aligned}
$$

with

$$
A=\sum_{n=1}^{k} C^{(n)} \lambda^{(n)}
$$

The extinction time $T_{\mathrm{ex}, 0}$ of $M_{t}$ is defined as

$$
T_{\mathrm{ex}, 0}=\inf \left\{t \mid \mathrm{E}\left[M_{t}\right]=0, t \geq 0\right\}
$$

which is exactly calculated as follows by (11). The extinction time $T_{\mathrm{ex}, 0}$ is calculated as

$$
T_{\mathrm{ex}, 0}=\frac{1}{R} \ln \left(1+\frac{M_{0}}{A}\right)=\frac{1}{R} \ln \left(1+M_{0}\left(\sum_{n=1}^{k} C^{(n)} \lambda^{(n)}\right)^{-1}\right) .
$$


Remark 3.1 The variance of the process $M_{t}$ is explicitly expressed for some $c_{t}^{(n)}$, although it has less simpler expression than that of the mean value (11). Assume that each $c_{t}^{(n)}$ follows a Cox-Ingersoll-Ross (CIR) model [32]

$$
\mathrm{d} c_{t}^{(n)}=a^{(n)}\left(C^{(n)}-c_{t}^{(n)}\right) \mathrm{d} t+\sigma^{(n)} \sqrt{c_{t}^{(n)}} \mathrm{d} B_{t}^{(n)}, \quad t>0,
$$

subject to the initial condition $c_{0}^{(n)}=C^{(n)}$ where $a^{(n)}>0$ and $\sigma^{(n)} \geq 0$, and $B_{t}^{(n)}$ (n= $1,2, \ldots, k)$ are standard 1-D Brownian motions independent with each other. For a positive initial condition, the process is strictly positive if [33]

$$
\sqrt{2 a^{(n)} C^{(n)}}>\sigma^{(n)}
$$

which is assumed throughout this paper. The CIR model is a minimal model that has a stationary distribution with exact mean and variance $[34,35]$. Assume that the process $c_{t}^{(n)}$ satisfies $c_{0}^{(n)}=C^{(n)}$. Then, the mean and variance of $c_{t}^{(n)}$ for $t>0$ are $\mathrm{E}\left[c_{t}^{(n)}\right]=C^{(n)}$ and $\operatorname{Var}\left[c_{t}^{(n)}\right]=\frac{C^{(n)}\left(\sigma^{(n)}\right)^{2}}{2 a^{(n)}}$, respectively. Actually, $c_{0}^{(n)}=C^{(n)}$ is a possible candidate of the initial condition, but may not be realized in practice. For the sake of simplicity of mathematical analysis, the mean value of the process $c_{t}^{(n)}$, which is weak stationary and has a finite invariant measure, can be approximated by $C^{(n)}$. A similar treatment is applied to the variance of $c_{t}^{(n)}$. This approximation does not significantly affect the numerical analysis results presented later because of the condition $a^{(n)} T \gg 1$ where the influences of the initial condition rapidly decay. By (16), the variance $\operatorname{Var}\left[c_{t}^{(n)}\right]$ is bounded from above as

$$
\operatorname{Var}\left[c_{t}^{(n)}\right]=\frac{C^{(n)}\left(\sigma^{(n)}\right)^{2}}{2 a^{(n)}}<\left(C^{(n)}\right)^{2}=\left(\mathrm{E}\left[c_{t}^{(n)}\right]\right)^{2} .
$$

The variance of the process $M_{t}$ is then exactly calculated with the help of the isometry formula for Poisson processes (Chapter 14 of [29]). The exact expression of the variance of $M_{t}$ is presented in Appendix 1, which is utilized in Section 4.

\subsection{Existence and uniqueness of optimal opening time}

Existence of the optimal opening time $\tau^{*}$, which is a maximizer of the performance index $J_{\tau}$, is mathematically analyzed. Several auxiliary results for analyzing $\tau^{*}$ are firstly presented. By (14), the extinction time $T_{\mathrm{ex}}=T_{\mathrm{ex}}(\tau)$ for $N_{t}$ is given as follows. The extinction time $T_{\text {ex }}=T_{\text {ex }}(\tau)$ for $N_{t}$ is

$$
T_{\mathrm{ex}}=\tau+\frac{1}{R} \ln \left(1+\frac{N_{\tau}}{B}\right)=\tau+\frac{1}{R} \ln \left(1+\frac{N_{0} \exp (-R \tau)}{B}\right) .
$$

A straightforward calculation shows that the first- and second-order derivatives of $T_{\mathrm{ex}}=$ $T_{\mathrm{ex}}(\tau)$ with respect to $\tau$ are

$$
\frac{\mathrm{d} T_{\mathrm{ex}}}{\mathrm{d} \tau}=\frac{B}{B+N_{0} \exp (-R \tau)}, \quad 0<\frac{\mathrm{d} T_{\mathrm{ex}}}{\mathrm{d} \tau}<1
$$

and

$$
\frac{\mathrm{d}^{2} T_{\mathrm{ex}}}{\mathrm{d} \tau^{2}}=R \frac{\mathrm{d} T_{\mathrm{ex}}}{\mathrm{d} \tau}\left(1-\frac{\mathrm{d} T_{\mathrm{ex}}}{\mathrm{d} \tau}\right)=\frac{B R N_{0} \exp (-R \tau)}{\left[B+N_{0} \exp (-R \tau)\right]^{2}}>0,
$$


respectively. Therefore, $T_{\mathrm{ex}}$ is convex and increasing with respect to $\tau$. Equation (19) leads to $T_{\mathrm{ex}}(\tau) \geq T_{\mathrm{ex}}(0)$ for $\tau \geq 0$, showing that $T_{\mathrm{ex}}(\tau) \geq T$ for $\tau \geq 0$ if $T_{\mathrm{ex}}(0) \geq T$. Therefore, $T_{\mathrm{ex}} \geq T$ if

$$
N_{0} \geq B(\exp (R T)-1)
$$

The inequality (21) means that there are remaining individuals at the terminal time $T$ when sufficiently large number of the individuals are introduced into the system.

It is shown below that behavior of the optimal opening time $\tau^{*}$ is qualitatively different between the exclusive cases $T_{\mathrm{ex}} \geq T$ and $T_{\mathrm{ex}}<T$. In both cases, the performance index $J_{\tau}$ can be rewritten as

$$
\begin{aligned}
J_{\tau}= & \int_{\tau}^{T \wedge T_{\mathrm{ex}}} \lambda^{(1)} \alpha^{(1)} C^{(1)} W_{t} \mathrm{~d} t+\int_{\tau}^{T \wedge T_{\mathrm{ex}}} \lambda^{(2)} \alpha^{(2)} C^{(2)} W_{t} \mathrm{~d} t \\
& -\int_{0}^{T \wedge T_{\mathrm{ex}}} \beta p \mathrm{E}\left[N_{t}\right] W_{t} \mathrm{~d} t \\
= & \tilde{A} \int_{\tau}^{T \wedge T_{\mathrm{ex}}} W_{t} \mathrm{~d} t-\beta p \int_{0}^{T \wedge T_{\mathrm{ex}}} \mathrm{E}\left[N_{t}\right] W_{t} \mathrm{~d} t
\end{aligned}
$$

with $\tilde{A}=\lambda^{(1)} \alpha^{(1)} C^{(1)}+\lambda^{(2)} \alpha^{(2)} C^{(2)}$. The first-order of partial derivative of $J_{\tau}$ with respect to $\tau$ is calculated as (Appendix 2)

$$
\frac{\mathrm{d} J_{\tau}}{\mathrm{d} \tau}=-\left[\tilde{A} W_{\tau}+\beta p B R \int_{\tau}^{T} \exp (-R(t-\tau)) W_{t} \mathrm{~d} t\right]<0
$$

for $T_{\mathrm{ex}} \geq T \cdot \frac{\mathrm{d} \tau}{\mathrm{d} \tau}$ is calculated as

$$
\frac{\mathrm{d} J_{\tau}}{\mathrm{d} \tau}=\tilde{A}\left(F_{\tau}-\eta G_{\tau}\right)
$$

for $T_{\text {ex }}<T$. Here,

$$
F_{\tau}=\frac{\mathrm{d} T_{\mathrm{ex}}}{\mathrm{d} \tau} W_{T_{\mathrm{ex}}}-W_{\tau}
$$

and

$$
G_{\tau}=\int_{\tau}^{T_{\mathrm{ex}}} \exp (-R(t-\tau)) W_{t} \mathrm{~d} t
$$

with $\eta=\frac{\beta p B R}{\tilde{A}}$.

The equation (23) immediately gives the following proposition since $J_{\tau}$ is monotonically decreasing with respect to $\tau$ when $T_{\mathrm{ex}} \geq T$. Define the critical opening time $\tau_{\mathrm{ex}}$ as

$$
\tau_{\text {ex }}=\inf \left\{\tau \geq 0 \mid T_{\text {ex }}(\tau) \geq T\right\} .
$$

The critical opening time $\tau_{\mathrm{ex}}$ is the unique positive solution to

$$
T=T_{\mathrm{ex}}\left(\tau_{\mathrm{ex}}\right)=\tau_{\mathrm{ex}}+\frac{1}{R} \ln \left(1+\frac{N_{0} \exp \left(-R \tau_{\mathrm{ex}}\right)}{B}\right)
$$


when the condition (21) does not hold, which certainly exists by (19) and (20). In addition, $0<\tau_{\mathrm{ex}}<T$. On the other hand, $\tau_{\mathrm{ex}}=0$ if (21). From a mathematical viewpoint, it is important to remark that $\tau^{*} \leq \tau_{\mathrm{ex}}$ when $T_{\mathrm{ex}} \geq T, J \in C\left(\left[0, T \wedge T_{\mathrm{ex}}\right]\right) \cap C^{2}\left(0, \tau_{\mathrm{ex}}\right) \cap C^{2}\left(\tau_{\mathrm{ex}}, T \wedge T_{\mathrm{ex}}\right)$, $\tau^{*}=0$ when (21). The condition $\tau^{*} \leq \tau_{\mathrm{ex}}$ when $T_{\mathrm{ex}} \geq T$ shows that it is optimal to set $\tau^{*}=0$ if $N_{0}$ is too large. Although this is theoretically optimal for such a case, it has not been employed in conventional aquacultures. Therefore, this strategy is not reasonable from the viewpoint of managing fishery resources. We thus assume that the condition (21) is not satisfied in what follows. The mathematical analysis below focus on the case $T_{\mathrm{ex}}<T$. The possible range of $\tau^{*}$ is then $\left[0, \tau_{\mathrm{ex}}\right]$. In the above-mentioned sense, $\tau_{\mathrm{ex}}$ is an upper bound of $\tau^{*}$.

A sufficient condition for $\tau^{*}>0$, which is consistent with the actual strategies for managing aquaculture systems by FCs, is presented below. Practical implications of the condition are presented as well.

Proposition $3.1 \tau^{*}>0$ if

$$
\frac{\mathrm{d} T_{\mathrm{ex}}(0)}{\mathrm{d} \tau} W_{T_{\mathrm{ex}}(0)}-W_{0}>\eta \int_{0}^{T_{\mathrm{ex}}(0)} \exp (-R t) W_{t} \mathrm{~d} t .
$$

Proof $\tau^{*}>0$ if $\left.\frac{\mathrm{d} \tau_{\tau}}{\mathrm{d} \tau}\right|_{\tau=0}>0$ since $J_{\tau}$ is continuously differentiable with respect to $\tau \geq 0$. Substituting (25) and (26) into $\left.\frac{\mathrm{d} \tau}{\mathrm{d} \tau}\right|_{\tau=0}>0$ leads to (29) since $\tilde{A}>0$.

Based on Proposition 3.1, the conditions to guarantee $\tau^{*}>0$ for several growth models are derived.

Proposition 3.2 For $g=r$ (= const) $>0$, the condition (29) is satisfied for sufficiently large $r$ and $\eta^{-1}$.

Proof The condition (29) is satisfied if its left-hand side is positive and $\eta$ is sufficiently small. The latter is always possible because the parameters $p, \beta, \alpha^{(1)}$, and $\alpha^{(2)}$ involved in $\eta$ do not appear in the left-hand side of (29). Actually, choosing sufficiently small $p \beta$ or sufficiently large $\tilde{A}=\lambda^{(1)} \alpha^{(1)} C^{(1)}+\lambda^{(2)} \alpha^{(2)} C^{(2)}$ can achieve arbitrary small and positive $\eta$.

Since $W_{t}=W_{0} \exp (r t)$ in the present case, the left-hand side of (29) becomes

$$
\frac{\mathrm{d} T_{\mathrm{ex}}(0)}{\mathrm{d} \tau} W_{T_{\mathrm{ex}}(0)}-W_{0}=\frac{B}{B+N_{0}} W_{T_{\mathrm{ex}}(0)}-W_{0}=\left[\left(1+\frac{N_{0}}{B}\right)^{\frac{r}{R}-1}-1\right] W_{0} .
$$

The right-hand side of (29) becomes

$$
\eta \int_{0}^{T_{\mathrm{ex}}(0)} \exp (-R t) W_{t} \mathrm{~d} t=\frac{\eta W_{0}}{r-R}\left(1-\exp \left(-R T_{\mathrm{ex}}(0)\right)\right)<\frac{\eta W_{0}}{r-R}
$$

if $r>R$. The condition (29) is thus satisfied if

$$
\left(1+\frac{N_{0}}{B}\right)^{\frac{r}{R}-1}>\frac{\eta W_{0}}{r-R}
$$

which holds true for sufficiently large $r$ and $\eta^{-1}$, which completes the proof. 
Proposition 3.2 then leads to the following theorem for the generalized Verhulst model:

$$
W_{t}=\left(\frac{K^{\theta}}{\left(K^{\theta} W_{0}^{-\theta}-1\right) \exp (-r \theta t)+1}\right)^{\frac{1}{\theta}}
$$

which behaves for sufficiently small $W_{0} K^{-1}$ as

$$
W_{t} \sim \frac{K}{\left(K^{\theta} W_{0}^{-\theta}-1\right)^{\frac{1}{\theta}}} \exp (r t)
$$

Theorem 3.1 For the generalized Verhulst model, the condition (29) is satisfied for sufficiently large $r$ and sufficiently small $W_{0} K^{-1}$ and $\eta$.

Proof Following the proof of Proposition 3.2, to prove the proposition, it is sufficient to show that the left-hand side of (29) can be made positive for sufficiently large $r$ and sufficiently small $K W_{0}^{-1}$. By (30) and (34), the left-hand side of (29) is evaluated as

$$
\frac{\mathrm{d} T_{\mathrm{ex}}(0)}{\mathrm{d} \tau} W_{T_{\mathrm{ex}}(0)}-W_{0} \sim \frac{K}{\left(K^{\theta} W_{0}^{-\theta}-1\right)^{\frac{1}{\theta}}}\left[\left(1+\frac{N_{0}}{B}\right)^{\frac{r}{R}-1}-1\right] .
$$

On the other hand, the right-hand side of (29) is evaluated as

$$
\eta \int_{0}^{T_{\mathrm{ex}}(0)} \exp (-R t) W_{t} \mathrm{~d} t<\frac{\eta K}{R}\left(1-\exp \left(-R T_{\mathrm{ex}}(0)\right)\right)<\frac{\eta K}{R} .
$$

Therefore, the condition (29) is satisfied if

$$
\frac{\eta}{R}<\frac{1}{\left(K^{\theta} W_{0}^{-\theta}-1\right)^{\frac{1}{\theta}}}\left[\left(1+\frac{N_{0}}{B}\right)^{\frac{r}{R}-1}-1\right]
$$

which holds true for sufficiently large $r$ and sufficiently small $W_{0} K^{-1}$ and $\eta$.

Remark 3.2 Proposition 3.2 and Theorem 3.1 show that the optimal opening time $\tau^{*}$ is positive, which is in accordance with the reality, if the aquacultured fishes grow sufficiently fast and large and the demand of harvesting them is sufficiently large. A practical implication of the analysis results on management of aquacultured fish is that the decision-maker should carefully observe their growth. Similar results have been theoretically derived in Yoshioka and Yaegashi [18] using a different population dynamics model based on an optimization approach. This fact implies that there exists an underlying universal principle for cost-effective management of aquacultured fish harvested at a certain rate after an opening time.

Uniqueness results of $\tau^{*}$ for small $p$ are finally presented in this sub-section. Key propositions for the case $p=0$, which seem to be not reasonable from a practical viewpoint but actually serve as the basis for dealing with the case with sufficiently small $p>0$, are presented below.

Proposition 3.3 For $p=0, g=r$ (= const $)>0$ and $r \neq R, \tau^{*} \in\left[0, \tau_{\mathrm{ex}}\right]$ exists uniquely. 
Proof By (24) and $\eta=0$,

$$
\frac{\mathrm{d} J_{\tau}}{\mathrm{d} \tau}=\tilde{A} F_{\tau}=\tilde{A}\left(\frac{\mathrm{d} T_{\mathrm{ex}}}{\mathrm{d} \tau} W_{T_{\mathrm{ex}}}-W_{\tau}\right), \quad 0 \leq \tau<\tau_{\mathrm{ex}} .
$$

By (18) and (19), (38) is rewritten as

$$
\frac{\mathrm{d} J_{\tau}}{\mathrm{d} \tau}=\tilde{A} W_{0} \frac{\mathrm{d} T_{\mathrm{ex}}}{\mathrm{d} \tau}\left[\exp \left((r-R)\left(T_{\mathrm{ex}}-\tau\right)\right)-1\right] \exp ((r-R) \tau), \quad 0 \leq \tau<\tau_{\mathrm{ex}},
$$

showing

$$
\operatorname{sgn}\left\{\frac{d J_{\tau}}{d \tau}\right\}=\operatorname{sgn}\{r-R\} .
$$

Therefore, by (23), $\tau^{*}$ is determined uniquely as

$$
\tau^{*}= \begin{cases}0 & (r<R), \\ \tau_{\mathrm{ex}} & (r>R) .\end{cases}
$$

Proposition 3.4 Assume $p=0, g$ is given by the generalized Verhulst model (2) with $r>R$ and $\theta \geq 1$. Then, $\tau^{*} \in\left[0, \tau_{\mathrm{ex}}\right]$ exists uniquely.

Proof In this case, $\eta=0$ as in Proposition 3.3 and

$$
\frac{\mathrm{d} J_{\tau}}{\mathrm{d} \tau}=\tilde{A} \frac{\mathrm{d} T_{\mathrm{ex}}}{\mathrm{d} \tau} \exp \left((r-R) T_{\mathrm{ex}}\right)\left[W_{T_{\mathrm{ex}}} \exp \left(-R T_{\mathrm{ex}}\right)-W_{\tau} \exp (-R \tau)\right], \quad 0 \leq \tau<\tau_{\mathrm{ex}}
$$

by (18) and (19). In addition,

$$
\frac{\mathrm{d}^{2} J_{\tau}}{\mathrm{d} \tau^{2}}<0
$$

when $\tau=\tau^{*} \in\left(0, \tau_{\mathrm{ex}}\right)$ exists (Proposition A.1 in Appendix 3). Therefore, $J_{\tau}$ does not have local minimum in $\left(0, \tau_{\text {ex }}\right)$. This follows that $\tau^{*} \in\left[0, \tau_{\text {ex }}\right]$ is determined uniquely with the help of the classical intermediate value theorem as

$$
\tau^{*}= \begin{cases}0 & \left(\text { if } F_{0} \leq 0\right), \\ \tilde{\tau} & \left(\text { if } F_{0}>0, F_{\tau_{\text {ex }}}<0\right), \\ \tau_{\text {ex }} & \left(\text { if } F_{0}>0, F_{\tau_{\text {ex }}} \geq 0\right),\end{cases}
$$

where $\tilde{\tau}$ is the unique solution of $F_{\tau}=0$.

Now, an application of the classical implicit function theorem with the smoothness of $F_{\tau}-\eta G_{\tau}$ with respect to $\tau$ and $p$ immediately shows the following unique existence theorem of $\tau^{*} \in\left(0, \tau_{\mathrm{ex}}\right)$ such that $\frac{\mathrm{d} \tau}{\mathrm{d} \tau}$ for small $p>0$.

Theorem 3.2 Assume (29). Then, under the assumption of Proposition 3.4 where ' $p=0$ ' is replaced by sufficiently small $p>0$ ', $\tau^{*} \in\left(0, \tau_{\mathrm{ex}}\right)$ exists uniquely. 
Theorem 3.2, although it can effectively characterize $\tau^{*} \in\left(0, \tau_{\mathrm{ex}}\right)$, is possibly not sharp as the numerical computation results demonstrate later.

\subsection{Comparative statics of the critical and optimal opening times}

Comparative statics of the critical opening time $\tau_{\text {ex }}$ and the optimal opening time $\tau^{*}$ such that $0<\tau^{*}<\tau_{\mathrm{ex}}$, namely their dependences on model parameters, is carried out. The comparative statics of $\tau_{\text {ex }}$ is firstly carried out. A straightforward calculation shows the following proposition.

Proposition 3.5 The critical opening time $\tau_{\mathrm{ex}}$ satisfies

$$
\begin{aligned}
\frac{\partial \tau_{\mathrm{ex}}}{\partial R} & =\frac{1}{R B}\left(T-\tau_{\mathrm{ex}}\right)\left[B+N_{0} \exp \left(-R \tau_{\mathrm{ex}}\right)\right]+\frac{N_{0} \tau_{\mathrm{ex}}}{R B} \exp \left(-R \tau_{\mathrm{ex}}\right)>0, \\
\frac{\partial \tau_{\mathrm{ex}}}{\partial N_{0}} & =-\frac{1}{R B} \exp \left(-R \tau_{\mathrm{ex}}\right)<0, \\
\frac{\partial \tau_{\mathrm{ex}}}{\partial C^{(n)}} & =\frac{N_{0} \lambda^{(n)}}{R B^{2}} \exp \left(-R \tau_{\mathrm{ex}}\right)>0 \quad(n=1,2),
\end{aligned}
$$

and

$$
\frac{\partial \tau_{\mathrm{ex}}}{\partial \lambda^{(n)}}=\frac{N_{0} C^{(n)}}{R B^{2}} \exp \left(-R \tau_{\mathrm{ex}}\right)>0 \quad(n=1,2) .
$$

Proof Partially differentiating both-hand sides of the governing equation of $\tau_{\mathrm{ex}}$, which is

$$
T=\tau_{\mathrm{ex}}+\frac{1}{R} \ln \left(1+\frac{N_{0} \exp \left(-R \tau_{\mathrm{ex}}\right)}{B}\right)
$$

with respect to $R$ leads to

$$
\begin{aligned}
0 & =\frac{\partial \tau_{\mathrm{ex}}}{\partial R}-\frac{1}{R^{2}} \ln \left(1+\frac{N_{0} \exp \left(-R \tau_{\mathrm{ex}}\right)}{B}\right)+\frac{1}{R} \frac{\partial}{\partial R} n\left(1+\frac{N_{0} \exp \left(-R \tau_{\mathrm{ex}}\right)}{B}\right) \\
& =\frac{\partial \tau_{\mathrm{ex}}}{\partial R}-\frac{1}{R}\left(T-\tau_{\mathrm{ex}}\right)+\frac{1}{R} \frac{N_{0} \frac{\partial}{\partial R} \exp \left(-R \tau_{\mathrm{ex}}\right)}{B+N_{0} \exp \left(-R \tau_{\mathrm{ex}}\right)} \\
& =\frac{B}{B+N_{0} \exp \left(-R \tau_{\mathrm{ex}}\right)} \frac{\partial \tau_{\mathrm{ex}}}{\partial R}-\frac{1}{R}\left(T-\tau_{\mathrm{ex}}\right)-\frac{1}{R} \frac{N_{0} \tau_{\mathrm{ex}} \exp \left(-R \tau_{\mathrm{ex}}\right)}{B+N_{0} \exp \left(-R \tau_{\mathrm{ex}}\right)},
\end{aligned}
$$

which is $(45)$ since $0<\tau_{\mathrm{ex}}<T$. Other inequalities are derived in a similar way.

Proposition 3.5 indicates that the critical opening time $\tau_{\mathrm{ex}}$ increases as the natural mortality rate of the fish $R$, the mean harvesting rates $C^{(n)}$, or the intensities $\lambda^{(n)}$. On the other hand, $\tau_{\mathrm{ex}}$ decreases as the initial total number of individuals $N_{0}$ increases. The results thus indicate that the critical opening time $\tau_{\mathrm{ex}}$, which is interpreted as an upper bound of the optimal opening time $\tau^{*}$, is larger (smaller) for relatively sparse (abundant) population in the aquaculture system.

Secondly, comparative statics of $\tau^{*}$ is carried out. Let $u$ be one of the parameters $p, \beta$, $\alpha^{(1)}$, or $\alpha^{(2)}$. Assume the interior solution $\tau^{*} \in\left(0, \tau_{\mathrm{ex}}\right)$. This $\tau^{*}$ solves

$$
F_{\tau^{*}}-\eta G_{\tau^{*}}=0 .
$$


In addition, assuming $\tau^{*}$ is an optimizer that gives a strict maximum of $J_{\tau}$ yields the second-order condition of optimality

$$
\frac{\mathrm{d}^{2} J_{\tau}}{\mathrm{d} \tau^{2}}=\left.\frac{\mathrm{d}\left(F_{\tau}-\eta G_{\tau}\right)}{\mathrm{d} \tau}\right|_{\tau=\tau^{*}}<0
$$

The following propositions reveal dependence of the above-mentioned $\tau^{*}$.

Proposition 3.6 Assume $\tau^{*} \in\left(0, \tau_{\mathrm{ex}}\right)$. Then, $\tau^{*}$ satisfies

$$
\frac{\partial \tau^{*}}{\partial u}=\frac{\partial \eta}{\partial u}\left(\left.\frac{\mathrm{d}^{2} J_{\tau}}{\mathrm{d} \tau^{2}}\right|_{\tau=\tau^{*}}\right)^{-1} G_{\tau^{*}}
$$

Proof Partially differentiating both-hand sides of (51) with respect to $u$ yields

$$
\begin{aligned}
0 & =\frac{\partial}{\partial u}\left(F_{\tau^{*}}-\eta G_{\tau^{*}}\right) \\
& =\left.\frac{\partial \tau^{*}}{\partial u} \frac{\mathrm{d}\left(F_{\tau^{*}}-\eta G_{\tau^{*}}\right)}{\mathrm{d} \tau}\right|_{\tau=\tau^{*}}-\frac{\partial \eta}{\partial u} G_{\tau^{*}} \\
& =\left.\frac{\partial \tau^{*}}{\partial u} \frac{\mathrm{d}^{2} J_{\tau}}{\mathrm{d} \tau^{2}}\right|_{\tau=\tau^{*}}-\frac{\partial \eta}{\partial u} G_{\tau^{*}},
\end{aligned}
$$

which is (53).

Proposition 3.6 leads to the following theorem.

Theorem 3.3 Assume $\tau^{*} \in\left(0, \tau_{\mathrm{ex}}\right)$. Then, $\tau^{*}$ satisfies

$$
\frac{\partial \tau^{*}}{\partial p}<0, \quad \frac{\partial \tau^{*}}{\partial \beta}<0, \quad \text { and } \quad \frac{\partial \tau^{*}}{\partial \alpha^{(n)}}>0 \quad(n=1,2) .
$$

Proof Substituting $\eta=\frac{\beta p B R}{\tilde{A}}$ and (52) into (53) with the positivity of $G_{\tau}$ leads to (55).

Theorem 3.3 has significant practical implications to management of aquacultured fish. Larger $p$ or $\beta$ gives smaller optimal opening time $\tau^{*}$, meaning that increasing the cost of farming the fishes makes the manager harvest them earlier. This is a consequence of the fact that the cost by farming, which is mainly for feeding the fishes and for cleaning up their excrements decreases as the total number of individuals decreases. On the other hand, larger $\alpha^{(1)}$ or $\alpha^{(2)}$ gives larger optimal opening time $\tau^{*}$. This implies that increasing the profit by harvesting the fishes, for sale and for exchange meetings for ecological education, makes the manager harvest them later. The aquacultured fishes are used for catching competition by children of local residents, and utility of the children can be larger with better-grown fishes since they can eat the caught fishes after the competition. Therefore, the dependence of $\tau^{*}$ on the parameters $\alpha^{(1)}$ or $\alpha^{(2)}$ is considered to be consistent with the reality. It should be noted that similar comparative statics results have been obtained in the deterministic optimization model for aquacultured fish [18]. 


\subsection{Analogy with a model for managing non-renewable released fish}

This sub-section shows an analogy between the present model for managing aquacultured fish and that for released fish, $P$. altivelis in particular. The fish has been the major inland fishery resource in Japan for recreational and fishery purposes. $P$. altivelis is an endemic fish in Japan and has an annual life history, which is reviewed in detail in the literature and the references therein [36-39]. In Japan, in each spring, FCs release juvenile P. altivelis into the rivers that they authorize. Harvesting the fish starts at the coming summer. The harvesting period is closed at the end of the autumn, on which the fishes spawn. The important point is that the released fish is often non-renewable possibly due to genetic and environmental reasons [40].

As a simple model we can hypothesize that the population dynamics of released $P$. altivelis in a river follows the SDEs (1) and (3) where the parameters and variables have different meanings. Under this setting, $R$ is the mortality rate of the fish by natural death and predation from waterfowls such as Phalacrocorax carbo [41]. The stochastic processes $P_{t}^{(1)}$ and $P_{t}^{(2)}$ represent the events of harvesting the fish for recreational and fishery purposes, respectively. We assume that the $\mathrm{FC}$ wants to choose the opening time of harvesting so that a performance index with the form (7) is maximized. The parameter $p$ in this case represents the cost of exterminating the predators; the parameters $R$ and $p$ may be dependent with each other. The weight parameters $\beta, \alpha^{(1)}$, and $\alpha^{(2)}$ depend on the attitude of the decision-maker as in the presented model for aquaculture. The above discussion implies that the present framework of mathematical modeling can be used not only for aquacultured fishes but also for some of the non-renewable released fishes.

\section{Results and discussion}

The present optimization model is applied to a demonstrative numerical computation of management of aquacultured $P$. altivelis.

\subsection{P. altivelis and HRFC}

In each year, farming juveniles of $P$. altivelis in an aquaculture system in Japan starts in spring (early to middle May) and they mature in summer around which harvesting opens (beginning of July). The harvesting ends in the coming autumn (late August to early October). HRFC in Shimane Prefecture, Japan farms $P$. altivelis from May to October in each year. Recently, officers of HRFC measured mean body weight of the individuals in the aquaculture system to track their growth. Both feeding the fish and cleaning up the pool are constantly carried out by the officers. According to the officers of HRFC, aquaculture of $P$. altivelis is one of the most indispensable sources of its income. They are trying to find cost-effective and ecologically sound management strategy of harvesting aquacultured $P$. altivelis.

\subsection{Parameter identification}

The previous research $[18,19]$ implied that the growth of P. altivelis in HRFC is reasonably described with the conventional Verhulst equation

$$
\frac{\mathrm{d} W_{t}}{\mathrm{~d} t}=r W_{t}\left(1-\frac{W_{t}}{K}\right)
$$




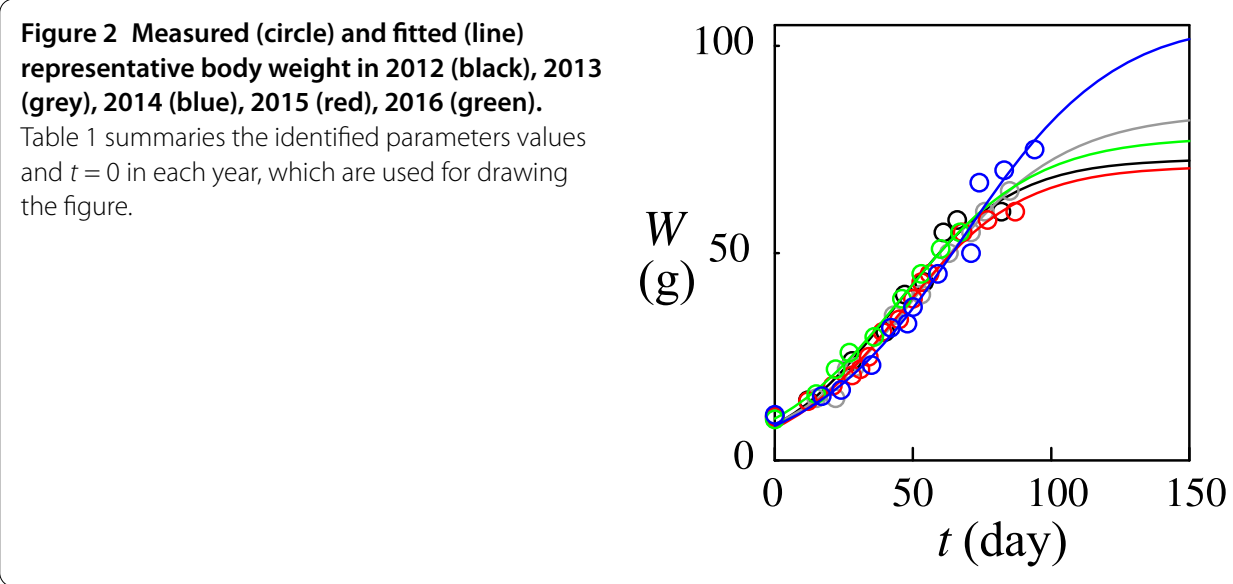

Table 1 The initial time $t=0$, the identified $W_{0}, K$, and $r$, and $\mathbf{R}^{2}$ value between the measured and identified $W_{t}$ in each year

\begin{tabular}{llrrll}
\hline Year & $\boldsymbol{t}=\mathbf{0}$ & $\boldsymbol{W}_{\mathbf{0}}(\mathbf{g})$ & $\boldsymbol{K}(\mathbf{g})$ & $\boldsymbol{r}(\mathbf{1} /$ day $)$ & $\mathbf{R}^{\mathbf{2}}$ \\
\hline 2012 & May 9 & 8.6 & 72.8 & 0.047 & 0.980 \\
2013 & May 9 & 8.7 & 83.8 & 0.040 & 0.995 \\
2014 & May 9 & 7.8 & 71.0 & 0.046 & 0.991 \\
2015 & May 7 & 10.1 & 78.0 & 0.042 & 0.997 \\
2016 & May 10 & 8.4 & 106.7 & 0.036 & 0.976 \\
\hline
\end{tabular}

Figure 2 shows the measured and fitted $W_{t}$ in each growth period from 2012 to 2016 . The circles are measured values and the curves are fitted growth curves based on the Verhulst model.

Table 1 summarizes the initial time $t=0$. The table also shows the identified values of the parameters $W_{0}, K$, and $r$ for each year using a nonlinear least squares method with the $\mathrm{R}^{2}$ value between the measured and identified $W_{t}$. The order of the parameters $W_{0}$, $K$, and $r$ are same in each year, while their values are slightly different between different years. Figure 2 and Table 1 show that the growth of aquacultured $P$. altivelis in each year is accurately approximated with the conventional Verhulst model (56). The identified result suggests $K=O\left(10^{2}\right)(\mathrm{g}), W_{0}=O\left(10^{\circ}\right)(\mathrm{g})$, and $r T=O\left(10^{\circ}\right)(-)$; the last one follows from the discussion below. In what follows the data in 2016 is used in the numerical computation as a demonstrative application of the present model.

The terminal time is set as $T=150$ (day) considering current management strategy by HRFC. The optimal opening time $\tau^{*}$ is computed directly from (51) using the classical Simpson's rule. The time increment for integration $\Delta \tau$ is set as 0.01 (day). The total number of individuals at the time $t=0$ is specified as $N_{0}=20,000$ based on an actual management strategy by HRFC. This $N_{0}$ has been empirically determined by HRFC considering the size of the pool used for their aquaculture.

The variance $\operatorname{Var}\left[N_{t}\right]$, which is an index used for verifying optimal management strategy, is obtained with the formula (69) in Appendix 1 as

$$
\operatorname{Var}\left[N_{t}\right]=\operatorname{Var}\left[M_{t-\tau}\right] \quad \text { with } k=2
$$

for $t \geq \tau$ and $\operatorname{Var}\left[N_{t}\right]=0$ for $0 \leq t<\tau$ with $M_{0}=N_{0}$. The standard deviation is then computed as $\sqrt{\operatorname{Var}\left[N_{t}\right]}$. The variance $\operatorname{Var}\left[N_{t}\right]$ is increasing with respect to each $\lambda^{(n)}, C^{(n)}$, and 
Figure 3 Optimal opening time $\tau^{*}$ in the $\boldsymbol{\alpha}^{(1)}-\boldsymbol{\alpha}^{(2)}$ 2-D parameter space. $\tau^{*}=0$ in the white area, which is placed near the origin $\left(\alpha^{(1)}, \alpha^{(2)}\right)=(0$, 0 ). The grey line corresponds to $\tau^{*}=52$ (day).

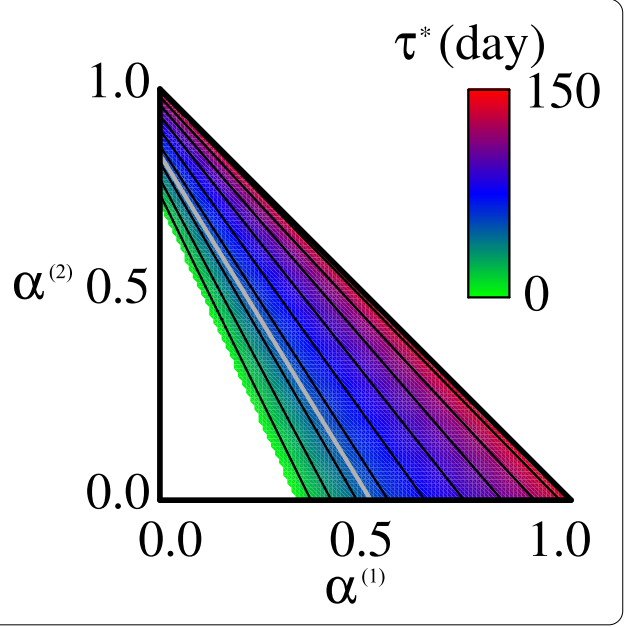

$\sigma^{(n)}$, while it is decreasing with respect to each $a^{(n)}$. Comparing the magnitude of the three terms of $\operatorname{Var}\left[N_{t}\right]$ (right-hand side of the formula (69) in Appendix 1) can quantify and qualify uncertainties involved in the management strategy.

Based on the personal communications with the officers of HRFC and their accounting book during 2016, the parameters $\lambda^{(n)}$ and $C^{(n)}$ are set as $\lambda^{(1)}=10\left(1 /\right.$ day), $\lambda^{(2)}=0.05$ $\left(1 /\right.$ day), $C^{(1)}=25(\mathrm{~kg}), C^{(2)}=1,000(\mathrm{~kg})$, respectively. The parameters $a^{(n)}$ and $\sigma^{(n)}$ are set as $a^{(1)}=a^{(2)}=1(1 /$ day $), \sigma^{(1)}=3(1 /$ day $)$, and $\sigma^{(2)}=5(1 /$ day $)$. The parameter $p$ is set as 0.001 (1/day). The mortality rate $R$ is set as 0.001 (1/day) considering the fact that almost $10 \%$ of aquacultured $P$. altivelis died during the growth period in 2016 . There are three weight parameters $\alpha^{(1)}, \alpha^{(2)}$, and $\beta$ in the model. Considering the form of the performance $\operatorname{index} J_{\tau}$, the following relationship is assumed without the loss of generality of analysis:

$$
\alpha^{(1)}+\alpha^{(2)}+\beta=1 \quad \text { with } \alpha^{(1)}, \alpha^{(2)}, \beta>0 .
$$

Therefore, choosing $\alpha^{(1)}$ and $\alpha^{(2)}$ gives $\beta$ as $\beta=1-\left(\alpha^{(1)}+\alpha^{(2)}\right)$.

\subsection{Computational results}

Figure 3 plots the optimal opening time $\tau^{*}$ in the 2-D $\alpha^{(1)}-\alpha^{(2)}$ parameter space. The grey line in the figure corresponds to $\tau^{*}$ by HRFC in 2016. In addition, Figure 4 plots the extinction time $T_{\text {ex }}$ computed with the optimal opening time $\tau^{*}$ in the 2-D $\alpha^{(1)}-\alpha^{(2)}$ parameter space. The grey line in the figure corresponds to $T_{\text {ex }}$ by HRFC in 2016. Figure 3 shows consistency between the computational result and the mathematical analysis result since there exists an area with $\tau^{*}=0$ near the origin $\left(\alpha^{(1)}, \alpha^{(2)}\right)=(0,0)$, at which $\eta$ is large and the inequality (21) is not satisfied. Figure 3 also shows that the computational results are consistent with the Theorem 3.2. The computational result suggests unique existence of $\tau^{*}$.

Conjecture 4.1 $\tau^{*} \in[0, T]$ exists uniquely.

Figure 5 plots $\mathrm{E}\left[N_{t}\right], \mathrm{E}\left[N_{t}\right] \pm \sqrt{\operatorname{Var}\left[N_{t}\right]}$, and ten sample paths of $N_{t}$ assuming the actual value $\tau^{*}=52$ (day) of HRFC in 2016 with the time increment for temporal integration 0.01 (day). The Runge-Kutta scheme [42] has been used for generating the sample paths where the transformation of variables $f_{t}=\sqrt{c_{t}^{(n)}}$, so that (15) is transformed to the SDE with the 

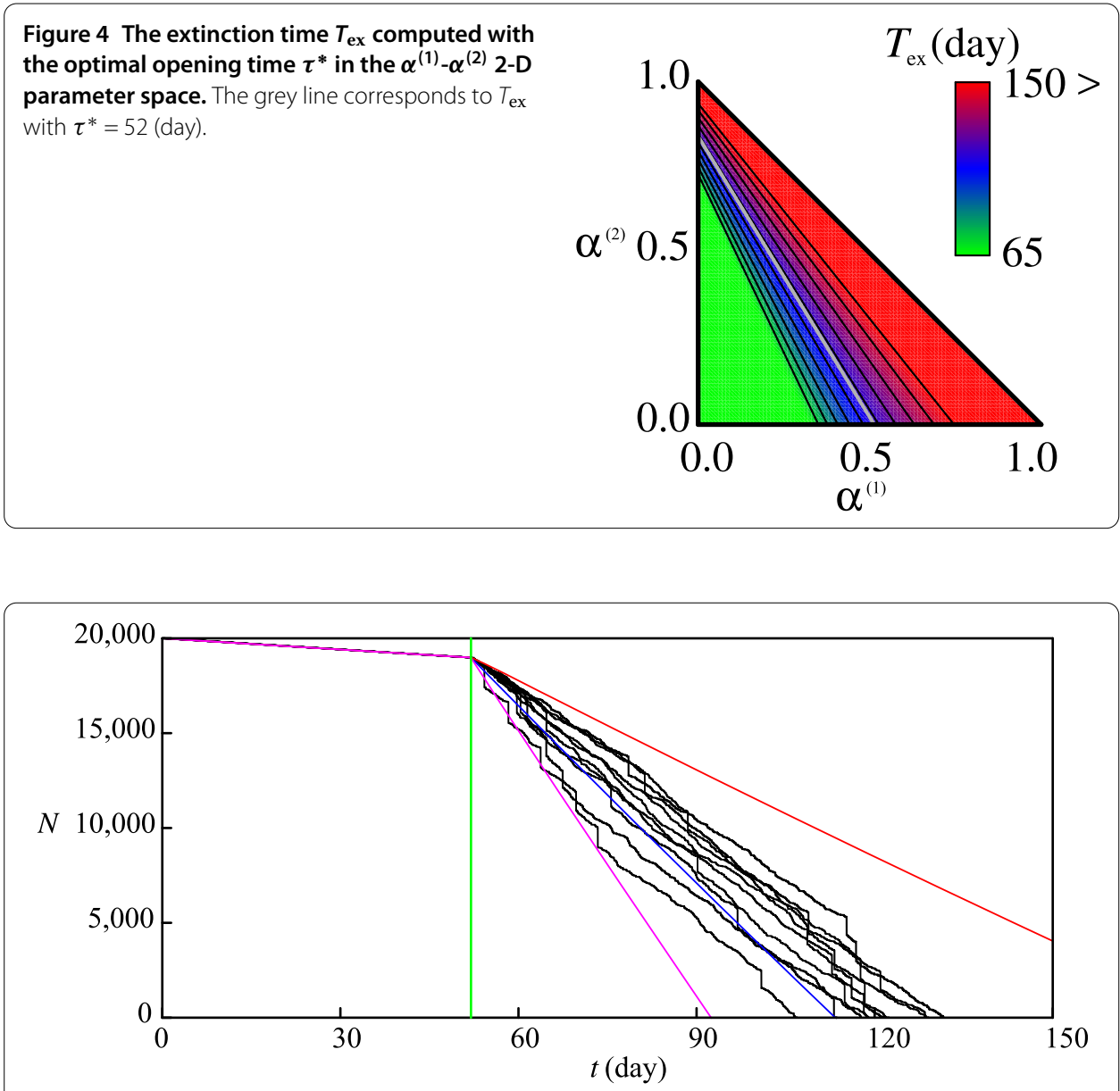

Figure $5 \mathrm{E}\left[N_{t}\right]$ (blue), $\mathrm{E}\left[N_{t}\right]+\sqrt{\operatorname{Var}\left[N_{t}\right]}$ (red), $\mathrm{E}\left[N_{t}\right]-\sqrt{\operatorname{Var}\left[N_{t}\right]}$ (pink), and sample paths of $N_{t}$ (black) assuming $\boldsymbol{\tau}^{*}=\mathbf{5 2}$ (green). Ten sample paths have been simulated using the strong numerical scheme (Wilkie [42]) with the time increment for temporal integration 0.01 (day).

additive noise term

$$
\mathrm{d} f_{t}=\frac{1}{2}\left[\frac{1}{f_{t}}\left(a^{(n)} C^{(n)}-\frac{\left(\sigma^{(n)}\right)^{2}}{4}\right)-a^{(n)} f_{t}\right] \mathrm{d} t+\frac{\sigma^{(n)}}{2} \mathrm{~d} B_{t}^{(n)}, \quad t>0,
$$

which is easier to numerically handle [33]. The sample paths of Figure 5 well characterize the behavior of $N_{t}$ that it is monotonically decreasing due to frequent small shocks by harvesting for sale and large and rare shocks for harvesting for ecological education, which is in accordance with the actual situation of HRFC. In the actual management strategy of P. altivelis by HRFC, $T_{\mathrm{ex}}$ in 2016 was 113 (day). The modelled extinction time $T_{\mathrm{ex}}$ with $\tau^{*}=52$ (day) is 113.4 (day), which agrees well with the actual value by HRFC in 2016, implying that the utilized parameter values are reasonable.

Finally, to compare impacts of the two different stochastic harvestings, the following stochastic variables that quantify the profit of the harvestings

$$
j^{(n)}=\int_{\tau^{*}}^{T \wedge T_{\mathrm{ex}}\left(\tau^{*}\right)} \alpha^{(n)} c_{t}^{(n)} W_{t} \mathrm{~d} P_{t}^{(n)} \quad(n=1,2)
$$




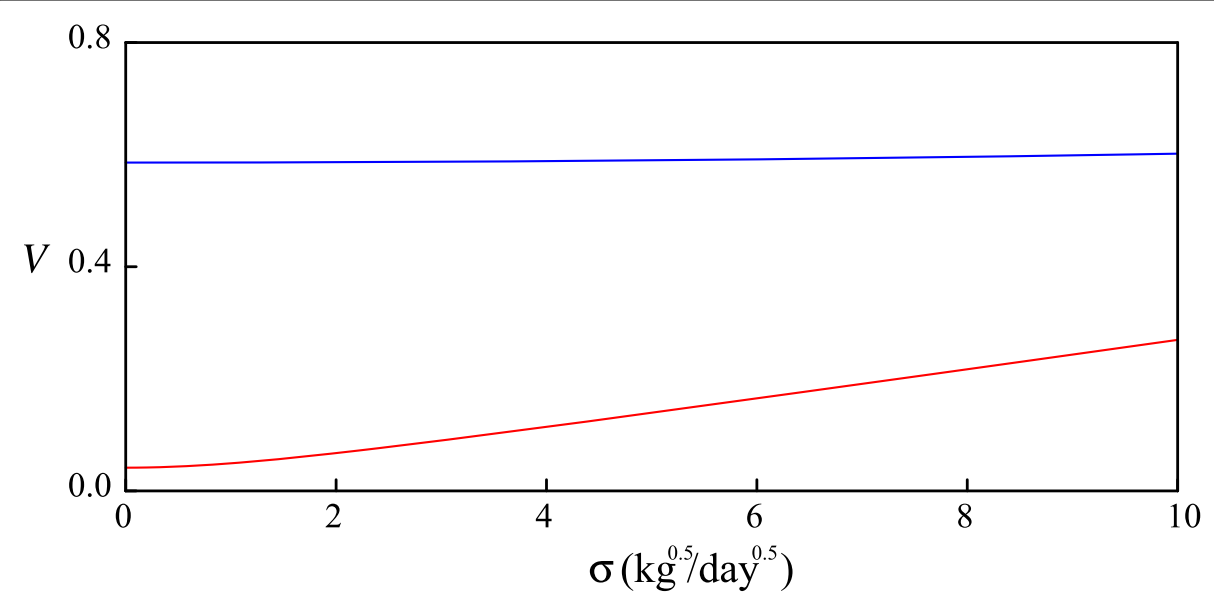

Figure 6 Numerically computed $V^{(n)}$ for $0 \leq \sigma^{(1)}, \sigma^{(2)} \leq 10\left(\mathrm{~kg}^{1 / 2} /\right.$ day $\left.^{1 / 2}\right)$ with $\tau^{*}=52$ (day): $n=1$ (harvesting for sale, red) and $n=2$ (harvesting for ecological education, blue).

are considered. The non-dimensional indexes to evaluate relative variability of the profits are presented as

$$
V^{(n)}=\frac{\sqrt{\operatorname{Var}\left[j^{(n)}\right]}}{\mathrm{E}\left[j^{(n)}\right]} \quad(n=1,2),
$$

which are computed with a numerical integration technique based on the standard midpoint rules. The index $V^{(n)}$ measures the fluctuation of the process $j_{\tau}^{(n)}$ relative to its mean value. Therefore, larger $V^{(n)}$ implies relatively larger stochastic fluctuations of $j^{(n)}$. The indexes $V^{(n)}$ are computed for different values of $\sigma^{(n)}$ to evaluate dependence of the profits by harvestings for sale and ecological education on the stochasticity of demands.

Figure 6 plots the numerically computed $V^{(n)}$ for $0 \leq \sigma^{(1)}, \sigma^{(2)} \leq 10\left(\mathrm{~kg}^{1 / 2} / \mathrm{day}^{1 / 2}\right)$. Figure 6 shows that the indexes $V^{(n)}$ are larger in the harvesting for ecological education $(n=2)$ than in the harvesting for sale $(n=1)$. In addition, each $V^{(n)}$ is monotonically increasing with respect to $\sigma^{(n)}$, and variability of $V^{(n)}$ for the range of $\sigma^{(n)}$ examined are larger in the harvesting for selling than in that for ecological education. The computational results show that the harvesting for sale is more robust against the stochastic fluctuation of the demand, implying that it can be a major source of profit of the FC. Nevertheless, the harvesting for ecological education, although it turned out to be less robust with larger $V^{(n)}$, actually should not be abandoned since it has served as an indispensable part of the work of the FC. The computational results in this paper provide useful information for decision-making on management of aquaculture systems subject to the stochasticity.

\section{Conclusion}

The present mathematical model is the first model that can evaluate the influences of the harvesting for ecological education on the aquaculture management. Mathematical and numerical analyses on the optimal opening time were carried out to comprehend its existence, uniqueness, and behavior. The analysis results focusing on P. altivelis in Japan demonstrated that the harvesting for ecological education is less robust than that for sale, but should not be abandoned since it has served as one of the most indispensable works of the FC. From the analysis results, we recommend FCs to create a working environment 
where the harvesting for ecological education can be more efficiently carried out with wider spillover effects. Financial and social supports from local governments would be helpful for FCs to achieve this attempt.

Future research will develop an optimization theory for long-term, multi-year management of aquacultured $P$. altivelis, so that FCs in Japan can find the way to achieve costeffective management strategy of the fish. In addition, local economic dynamics around the FCs will also be mathematically described, so that the demand of the fishes is more reasonably tracked. Investigations both from theoretical and practical viewpoints are necessary for further advance of fishery science and engineering.

\section{Appendix 1}

This appendix presents a calculation procedure of the variance of $M_{t}$, which is defined as $\operatorname{Var}\left[M_{t}\right]=\mathrm{E}\left[M_{t}^{2}\right]-\left(\mathrm{E}\left[M_{t}\right]\right)^{2}$. By the martingale property of the compensated Poisson process $P_{t}^{(n)}-\lambda^{(n)} t$ (Chapter 15 of [29]) and stationarity of $c_{t}^{(n)}, \mathrm{E}\left[M_{t}^{2}\right]$ satisfies

$$
\begin{aligned}
& \exp (2 R t) \mathrm{E}\left[M_{t}^{2}\right] \\
& =\mathrm{E}\left[\left(M_{0}-\int_{0}^{t} \exp (R s) \sum_{n=1}^{k} c_{s}^{(n)} \mathrm{d} P_{s}^{(n)}\right)^{2}\right] \\
& =M_{0}^{2}-2 M_{0} B(\exp (R t)-1)+\mathrm{E}\left[\left(\int_{0}^{t} \exp (R s) \sum_{n=1}^{k} \lambda^{(n)} c_{s}^{(n)} \mathrm{d} s\right)^{2}\right] \\
& \quad+\mathrm{E}\left[\sum_{n=1}^{k} \lambda^{(n)} \int_{0}^{t}\left(c_{s}^{(n)}\right)^{2} \exp (2 R s) \mathrm{d} s\right] .
\end{aligned}
$$

By stationarity and independency of $c_{t}^{(n)}$, the second term in the right-hand side of (62) becomes

$$
\begin{aligned}
\mathrm{E} & {\left[\left(\int_{0}^{t} \exp (R s) \sum_{n=1}^{k} \lambda^{(n)} c_{s}^{(n)} \mathrm{ds}\right)^{2}\right] } \\
= & \sum_{m=1}^{k} \sum_{n=1}^{k} \lambda^{(n)} \lambda^{(m)} \int_{0}^{t} \int_{0}^{t} \exp (R(s+u)) \mathrm{E}\left[c_{u}^{(m)} c_{s}^{(n)}\right] \mathrm{d} s \mathrm{~d} u \\
= & \sum_{m \neq n, m \geq 1, n \geq 1}^{k} \lambda^{(m)} \lambda^{(n)} C^{(n)} C^{(m)}\left(\frac{\exp (R t)-1}{R}\right)^{2} \\
& +\sum_{n=1}^{k}\left(\lambda^{(n)}\right)^{2} \int_{0}^{t} \int_{0}^{t} \exp (R(s+u)) \mathrm{E}\left[c_{u}^{(n)} c_{s}^{(n)}\right] \mathrm{d} s \mathrm{~d} u .
\end{aligned}
$$

Then, (62) is rewritten as

$$
\begin{aligned}
\exp (2 R t) \mathrm{E}\left[M_{t}^{2}\right]= & \exp (2 R t)\left(\mathrm{E}\left[M_{t}\right]\right)^{2}-B^{2}(\exp (R t)-1)^{2} \\
& +\sum_{m \neq n, m \geq 1, n \geq 1}^{k} \lambda^{(m)} \lambda^{(n)} C^{(n)} C^{(m)}\left(\frac{\exp (R t)-1}{R}\right)^{2}
\end{aligned}
$$




$$
\begin{aligned}
& +\sum_{n=1}^{k}\left(\lambda^{(n)}\right)^{2} \int_{0}^{t} \int_{0}^{t} \exp (R(s+u)) \mathrm{E}\left[c_{u}^{(n)} c_{s}^{(n)}\right] \mathrm{d} s \mathrm{~d} u \\
& +\mathrm{E}\left[\sum_{n=1}^{k} \lambda^{(n)} \int_{0}^{t}\left(c_{s}^{(n)}\right)^{2} \exp (2 R s) \mathrm{d} s\right] .
\end{aligned}
$$

Note that

$$
\begin{aligned}
& \mathrm{E}\left[\sum_{n=1}^{k} \int_{0}^{t}\left(c_{s}^{(n)}\right)^{2} \exp (2 R s) \mathrm{d} s\right]=\frac{(\exp (R t)-1)(\exp (R t)+1)}{4 R} \sum_{n=1}^{k} \frac{C^{(n)}\left(\sigma^{(n)}\right)^{2}}{a^{(n)}}, \\
& \mathrm{E}\left[c_{u}^{(n)} c_{s}^{(n)}\right]=\frac{C^{(n)}\left(\sigma^{(n)}\right)^{2}}{2 a^{(n)}} \exp \left(-a^{(n)}|u-s|\right)+\left(C^{(n)}\right)^{2}, \\
& \sum_{n=1}^{k}\left(\lambda^{(n)}\right)^{2} \int_{0}^{t} \int_{0}^{t} \exp (R(s+u-2 t)) \mathrm{E}\left[c_{u}^{(n)} c_{s}^{(n)}\right] \mathrm{d} s \mathrm{~d} u \\
& =B^{2}(1-\exp (-R t))^{2} \\
& \quad+\sum_{n=1}^{k} \frac{\left(\lambda^{(n)}\right)^{2} C^{(n)}\left(\sigma^{(n)}\right)^{2}}{2 a^{(n)}} \int_{0}^{t} \int_{0}^{t} \exp \left(R(s+u-2 t)-a^{(n)}|u-s|\right) \mathrm{d} s \mathrm{~d} u,
\end{aligned}
$$

and

$$
\begin{aligned}
& \int_{0}^{t} \int_{0}^{t} \exp \left(R(s+u-2 t)-a^{(n)}|u-s|\right) \mathrm{d} s \mathrm{~d} u \\
& \quad=\frac{2 \exp (-2 R t)}{R+a^{(n)}} \begin{cases}\frac{\exp (2 R t)-1}{2 R}-t & \left(R=a^{(n)}\right), \\
\frac{\exp (2 R t)-1}{2 R}-\frac{\exp \left(\left(R-a^{(n)}\right) t\right)-1}{R-a^{(n)}} & \left(R \neq a^{(n)}\right) .\end{cases}
\end{aligned}
$$

Therefore, with (65) through (68), a closed-form expression of the variance $\operatorname{Var}\left[M_{t}\right]$ is obtained as

$$
\begin{aligned}
\operatorname{Var}\left[M_{t}\right]= & \sum_{m \neq n, m \geq 1, n \geq 1}^{k} \lambda^{(m)} \lambda^{(n)} C^{(n)} C^{(m)}\left(\frac{1-\exp (-R t)}{R}\right)^{2} \\
& +\sum_{n=1}^{k} \frac{\left(\lambda^{(n)}\right)^{2} C^{(n)}\left(\sigma^{(n)}\right)^{2}}{2 a^{(n)}} \\
& \times \int_{0}^{t} \int_{0}^{t} \exp \left(R(s+u-2 t)-a^{(n)}|u-s|\right) \mathrm{d} s \mathrm{~d} u \\
& +\frac{(1+\exp (-R t))(1-\exp (-R t))}{2 R} \sum_{n=1}^{k} \frac{\lambda^{(n)} C^{(n)}\left(\sigma^{(n)}\right)^{2}}{a^{(n)}} .
\end{aligned}
$$

The formula (69) shows that $\operatorname{Var}\left[M_{t}\right]$ is increasing with respect to each $\lambda^{(n)}, C^{(n)}$, and $\sigma^{(n)}$, while it is decreasing with respect to each $a^{(n)}$.

\section{Appendix 2}

This appendix shows calculation procedures of the first-order derivative of $J_{\tau}$. A key of the calculation procedures is using the Leibnitz's rule (Appendix A of [43]). By (5), assuming 
$T>T_{\text {ex }}$, differentiating both-hand sides of (22) with respect to $\tau$ yields

$$
\begin{aligned}
\frac{\mathrm{d} J_{\tau}}{\mathrm{d} \tau} & =\frac{\mathrm{d}}{\mathrm{d} \tau}\left[\tilde{A} \int_{\tau}^{T} W_{t} \mathrm{~d} s-\beta p \int_{0}^{T} \mathrm{E}\left[N_{t}\right] W_{t} \mathrm{~d} t\right] \\
& =-\tilde{A} W_{\tau}-\beta p N_{0} \exp (-R \tau) W_{\tau}-\beta p \frac{\mathrm{d}}{\mathrm{d} \tau} \int_{\tau}^{T} \mathrm{E}\left[N_{t}\right] W_{t} \mathrm{~d} t .
\end{aligned}
$$

Since

$$
\begin{aligned}
- & \beta p \frac{\mathrm{d}}{\mathrm{d} \tau} \int_{\tau}^{T} \mathrm{E}\left[N_{t}\right] W_{t} \mathrm{~d} t \\
& =\beta p\left(N_{0} \exp (-R \tau) W_{\tau}-B R \int_{\tau}^{T} \exp (-R(t-\tau)) W_{t} \mathrm{~d} t\right),
\end{aligned}
$$

combining (70) and (71) yields

$$
\frac{\mathrm{d} J_{\tau}}{\mathrm{d} \tau}=-\tilde{A} W_{\tau}-\beta p B R \int_{\tau}^{T} \exp (-R(t-\tau)) W_{t} \mathrm{~d} t
$$

which is (23). Similarly, for $T>T_{\mathrm{ex}}$,

$$
\frac{\mathrm{d} J_{\tau}}{\mathrm{d} \tau}=\frac{\mathrm{d}}{\mathrm{d} \tau}\left[\tilde{A} \int_{\tau}^{T_{\mathrm{ex}}} W_{t} \mathrm{~d} s-\beta p \int_{0}^{T_{\mathrm{ex}}} \mathrm{E}\left[N_{t}\right] W_{t} \mathrm{~d} t\right] .
$$

The first term in the right-hand side of (73) is calculated as

$$
\tilde{A} \frac{\mathrm{d}}{\mathrm{d} \tau} \int_{\tau}^{T_{\mathrm{ex}}} W_{t} \mathrm{~d} s=\tilde{A}\left(\frac{\mathrm{d} T_{\mathrm{ex}}}{\mathrm{d} \tau} W_{T_{\mathrm{ex}}}-W_{\tau}\right)=\tilde{A} F_{\tau}
$$

By (71), the second term in the right-hand side of (73) is calculated as

$$
\begin{aligned}
\frac{\mathrm{d}}{\mathrm{d} \tau}\left[-\beta p \int_{0}^{T_{\mathrm{ex}}} \mathrm{E}\left[N_{t}\right] W_{t} \mathrm{~d} t\right]= & -\beta p \frac{\mathrm{d}}{\mathrm{d} \tau}\left[\int_{0}^{\tau} \mathrm{E}\left[N_{t}\right] W_{t} \mathrm{~d} t+\int_{\tau}^{T_{\mathrm{ex}}} \mathrm{E}\left[N_{t}\right] W_{t} \mathrm{~d} t\right] \\
= & -\beta p\left(\mathrm{E}\left[N_{\tau}\right] W_{\tau}+\frac{\mathrm{d} T_{\mathrm{ex}}}{\mathrm{d} \tau} \mathrm{E}\left[N_{T_{\mathrm{ex}}}\right] W_{T_{\mathrm{ex}}}\right. \\
& \left.-N_{0} \exp (-R \tau) W_{\tau}+\int_{\tau}^{T_{\mathrm{ex}}} \frac{\mathrm{d}}{\mathrm{d} \tau} \mathrm{E}\left[N_{t}\right] W_{t} \mathrm{~d} t\right) \\
= & -\tilde{A} \eta G_{\tau}
\end{aligned}
$$

since $\mathrm{E}\left[N_{T_{\mathrm{ex}}}\right]=0$. Combining (73), (74), and (75) yields (24).

\section{Appendix 3}

The following proposition used in Proposition 3.4 is proven in this appendix.

Proposition A.1 Assume $p=0$ and $g$ is given by the generalized Verhulst model (2) with $r>R$ and $\theta \geq 1$. In addition, assume $\tau^{*} \in\left(0, \tau_{\mathrm{ex}}\right)$ solves $\frac{\mathrm{d} J_{\tau}}{\mathrm{d} \tau}=0$. Then, $\frac{\mathrm{d}^{2} \tau_{\tau}}{\mathrm{d} \tau^{2}}<0$ at $\tau=\tau^{*}$. 
Proof Under the stated assumption, $\frac{\mathrm{d}^{2} J}{\mathrm{~d} \tau^{2}}$ at $\tau=\tau^{*}$ is

$$
\frac{1}{\tilde{A}} \frac{\mathrm{d}^{2} J_{\tau}}{\mathrm{d} \tau^{2}}=\left(\frac{\mathrm{d} T_{\mathrm{ex}}}{\mathrm{d} \tau}\right)^{2} g\left(W_{T_{\mathrm{ex}}}\right) W_{T_{\mathrm{ex}}}+\frac{\mathrm{d}^{2} T_{\mathrm{ex}}}{\mathrm{d} \tau^{2}} W_{T_{\mathrm{ex}}}-g\left(W_{\tau}\right) W_{\tau}
$$

which can be rewritten with (20) as

$$
\frac{1}{\tilde{A}} \frac{\mathrm{d}^{2} J_{\tau}}{\mathrm{d} \tau^{2}}=\frac{W_{\tau}}{W_{T_{\mathrm{ex}}}}\left[\left(R-g\left(W_{\tau}\right)\right) W_{T_{\mathrm{ex}}}+\left(g\left(W_{T_{\mathrm{ex}}}\right)-R\right) W_{\tau}\right] \quad \text { at } \tau=\tau^{*} .
$$

Substituting (2) into (77) yields

$$
\begin{aligned}
\frac{1}{\tilde{A}} \frac{\mathrm{d}^{2} J_{\tau}}{\mathrm{d} \tau^{2}} & =\frac{W_{\tau}}{W_{T_{\mathrm{ex}}}}\left[-(r-R)\left(W_{T_{\mathrm{ex}}}-W_{\tau}\right)-r K^{-\theta} W_{\tau} W_{T_{\mathrm{ex}}}\left(W_{T_{\mathrm{ex}}}^{\theta-1}-W_{\tau}^{\theta-1}\right)\right] \\
& <0 \quad \text { at } \tau=\tau^{*},
\end{aligned}
$$

which with $\tilde{A}>0$ proves the proposition.

\section{Acknowledgements}

The River Fund No. 285311020 in charges of The River Foundation, JSPS Research Grant Nos. 15H06417, 17K15345, and WEC Applied Ecology Research Grant No. 2016-02 support this research. The authors thank to Hii River Fishery Cooperatives for providing valuable data.

\section{Funding}

The River Fund No. 285311020 in charges of The River Foundation, JSPS Research Grant Nos. 15H06417, 17K15345, and WEC Applied Ecology Research Grant No. 2016-02 support this research.

\section{Competing interests}

The authors declare that they have no competing interests.

\section{Authors' contributions}

HY wrote the paper with the support of YY. HY and YY performed mathematical and numerical analysis, and interviews to officers of Hii River Fisheries Cooperatives. All authors read and approved the final manuscript.

\section{Authors' information}

Yuta Yaegashi is Research Fellow of Japan Society for the Promotion of Science.

\section{Author details}

${ }^{1}$ Faculty of Life and Environmental Science, Shimane University, Nishikawatsu-cho 1060, Matsue, Shimane 690-8504, Japan. ${ }^{2}$ Graduate School of Agriculture, Kyoto University, Kitashirakawa-oiwake-cho, Sakyo-ku, Kyoto, Kyoto 606-8502, Japan.

\section{Publisher's Note}

Springer Nature remains neutral with regard to jurisdictional claims in published maps and institutional affiliations.

Received: 13 March 2017 Accepted: 11 May 2017 Published online: 19 May 2017

\section{References}

1. Gjedrem T, Robinson N, Rye M. The importance of selective breeding in aquaculture to meet future demands for animal protein: a review. Aquaculture. 2012;350:117-29. doi:10.1016/j.aquaculture.2012.04.008.

2. Clarke JL, Waheed MT, Lössl AG, Martinussen I, Daniell H. How can plant genetic engineering contribute to cost-effective fish vaccine development for promoting sustainable aquaculture? Plant Mol Biol. 2013;83:33-40. doi:10.1007/s11103-013-0081-9.

3. Mazid MA, Zaher M, Begum NN, Ali MZ, Nahar F. Formulation of cost-effective feeds from locally available ingredients for carp polyculture system for increased production. Aquaculture. 1997;151:71-8. doi:10.1016/S0044-8486(96)01504-9.

4. Myers RA, Barrowman NJ, Hutchings JA, Rosenberg AA. Population dynamics of exploited fish stocks at low population levels. Science. 1995;269(5227):1106. doi:10.1126/science.269.5227.1106.

5. Victor BC. Recruitment and population dynamics of a coral reef fish. Science. 1983;219(4583):419-20. doi:10.1126/science.219.4583.419.

6. Schaefer MB. Some considerations of population dynamics and economics in relation to the management of the commercial marine fisheries. J Fish Res Board Can. 1957;14:669-81. doi:10.1139/f57-025. 
7. Frigessi A, Holden L, Teigland A. (sfi) ${ }^{2}$ statistics for innovation - the experience of the Oslo centre in industrial statistics. Appl Stoch Models Bus Ind. 2015;31:82-93. doi:10.1002/asmb.2113.

8. Llorente I, Luna L. Bioeconomic modelling in aquaculture: an overview of the literature. Aquac Int. 2015;24:1-18. doi:10.1007/s10499-015-9962-z.

9. McCausland WD, Mente E, Pierce GJ, Theodossiou I. A simulation model of sustainability of coastal communities: aquaculture, fishing, environment and labour markets. Ecol Model. 2006;193:271-94. doi:10.1016/j.ecolmodel.2005.08.028.

10. Sana SS. Optimal pricing strategy for livestock of fishery and poultry. Econ Model. 2012;29:1024-34. doi:10.1016/j.econmod.2012.03.010.

11. Schechtman E, Wax $Y$, Tandler A. The effect of photoperiod and prey density on long-term survival of larvae - a data analytic approach where no parametric model is assumed and individual follow-up is not available. Appl Stoch Models Data Anal. 1990;6:27-40. doi:10.1002/asm.3150060104.

12. Øksendal B. Stochastic differential equations. Berlin: Springer; 2003.

13. Nøstbakken L. Regime switching in a fishery with stochastic stock and price. J Environ Econ Manag. 2006;51:231-41. doi:10.1016/j.jeem.2005.07.001.

14. Reed WJ, Clarke HR. Harvest decisions and asset valuation for biological resources exhibiting size-dependent stochastic growth. Int Econ Rev. 1990;31:147-69. doi:10.2307/2526634.

15. León-Santana M, Hernández JM. Optimum management and environmental protection in the aquaculture industry. Ecol Econ. 2008;64:849-57. doi:10.1016/j.ecolecon.2007.05.006.

16. Grantham RW, Rudd MA. Current status and future needs of economics research of inland fisheries. Fisheries Manag Ecol. 2015;22:458-71. doi:10.1111/fme.12144.

17. Welcomme RL, Cowx IG, Coates D, Béné C, Funge-Smith S, Halls A, Lorenzen K. Inland capture fisheries. Philos Trans R Soc Lond B, Biol Sci. 2010;365:2881-96. doi:10.1098/rstb.2010.0168.

18. Yoshioka $\mathrm{H}$, Yaegashi Y. Finding the optimal opening time of harvesting farmed fishery resources. Pac J Math Ind. 2016;8:6. doi:10.1186/s40736-016-0025-9.

19. Yoshioka H, Yaegashi Y. Optimal criteria to start harvesting in stochastic aquaculture system. Appl Stoch Models Bus Ind. In press 2017. doi:10.1002/asmb.2250.

20. Dixit AK, Pindyck RS. Investment under uncertainty. Princeton: Princeton University Press; 1994.

21. Abe S, Sajiki T, Tamaki Y, Awata S, Iguchi K. Educational significance of fishermen's activities providing opportunities for schoolchildren to play in streams. Jpn J Limnol (Rikusuigaku Zasshi). 2013;74:165-71. doi:10.3739/rikusui.74.165 (in Japanese with English abstract).

22. Oshitani M, Seki I, Hayashi H, Nishizaki T. The actual state of exchange between urban resident and fishing community and a subject of vitalization of region. Proc Civ Eng Ocean. 2004;20:311-5 (in Japanese with English abstract).

23. Japan Fisheries Agency. http://www.jfa.maff.go.jp/j/enoki/naisuimeninfo.html (2017). Accessed 1 Jan 2017.

24. Hii River Fisheries Cooperative. Se-oto (Annual public relations magazine published by HRFC), p. 5. http://www.hiikawafish.jp/date/160701_seoto_no9.pdf. Accessed 28 Dec 2016.

25. Blanchard EA, Loxton R, Rehbock V. A computational algorithm for a class of non-smooth optimal control problems arising in aquaculture operations. Appl Math Comput. 2013;219:8738-46. doi:10.1016/j.amc.2013.02.070.

26. Neubert MG. Marine reserves and optimal harvesting. Ecol Lett. 2003;6:843-9. doi:10.1046/j.1461-0248.2003.00493.x

27. Pascoe S, Wattage P, Naik D. Optimal harvesting strategies: practice versus theory. Aquac Econ Manag. 2002;6:295-308. doi:10.1080/13657300209380320.

28. Privault N. Notes on Stochastic Finance. https://drive.google.com/file/d/OB29QNyowSj9sM210Z2RaMG41VFE/edit (2014). Accessed 28 Dec 2016.

29. Ross SM. Applied probability models with optimization applications. New York: Dover; 2013.

30. Thieme HR. Mathematics in population biology. Princeton: Princeton University Press; 2003.

31. Strzałka D, Grabowski F. Towards possible $q$-generalizations of the Malthus and Verhulst growth models. Phys A, Stat Mech Appl. 2008;387:2511-8. doi:10.1016/.jphysa.2007.12.014.

32. Cox JC, Ingersoll JE, Ross SA. Theory of the term structure of interest rates. Econometrica. 1985;53:385-407. doi:10.2307/1911242.

33. Platen E, Bruti-Liberati N. Numerical solution of stochastic differential equations with jumps in finance. Heidelberg: Springer; 2010. p. 62.

34. Bensoussan A, Brouste A. Cox-Ingersoll-Ross model for wind speed modeling and forecasting. Wind Energy. 2015;19:1355-65. doi:10.1002/we.1896.

35. Campillo F, Joannides M, Larramendy-Valverde I. Stochastic modeling of the chemostat. Ecol Model. 2011;222:2676-89. doi:10.1016/j.ecolmodel.2011.04.027.

36. MAFF. Statistics agriculture, forestry and fisheries in Japan during 2014 (in Japanese). http://www.maff.go.jp/j/tokei/kouhyou/kaimen_gyosei/pdf/gyogyou_seisan_14.pdf. Accessed 25 Jun 2016.

37. Katano $\mathrm{O}$, Abe S, Nakamura T. Relationships between ayu Plecoglossus altivelis altivelis and other organisms in stream communities. Bull Fish Res Agency. 2006;Suppl 5:203-8 (in Japanese with English abstract).

38. Takahashi I, Azuma K. The up-to-now knowledge book of ayu. Tokyo: Tsukiji-shokan; 2006 (in Japanese).

39. Tanaka Y, Iguchi K, Yoshimura J, Nakagiri N, Tainaka K. Historical effect in the territoriality of ayu fish. J Theor Biol. 2011;268:98-104. doi:10.1016/j.jtbi.2010.09.040.

40. Tago Y. Survival of a landlocked form of ayu fry released in the Shou River. Aquac Sci. 1999;47:111-2. doi:10.11233/aquaculturesci1953.47.111 (in Japanese).

41. Kumada N, Arima T, Tsuboi II, Ashizawa A, Fujioka M. The multi-scale aggregative response of cormorants to the mass stocking of fish in rivers. Fish Res. 2013;137:81-7. doi:10.1016/j.fishres.2012.09.005.

42. Wilkie J. Numerical methods for stochastic differential equations. Phys Rev E. 2004;70:017701. doi:10.1103/PhysRevE.70.017701.

43. Yoshioka $\mathrm{H}$, Unami $\mathrm{K}$. A cell-vertex finite volume scheme for solute transport equations in open channel networks. Probab Eng Mech. 2013;31:30-8. doi:10.1016/.jprobengmech.2012.12.001. 